\title{
Linear Viscoelastic Properties of Polybutadiene. A Comparison With Molecular Theories
}

\author{
Jacques ROOVERS \\ Division of Chemistry, National Research Council of Canada, \\ Ottawa, Ontario, Canada K1A OR9
}

(Received August 15, 1985)

\begin{abstract}
The linear viscoelastic properties of a series of linear entangled polybutadienes were studied. It is found that the melt viscosity $\eta_{0} \propto M^{3.38}$ and the plateau modulus $G_{\mathrm{N}}^{\circ} \propto M^{\circ}$. The zero-shear recoverable compliance $J_{\mathrm{e}}^{\circ}$ decreases slightly with increasing MW above 40,000. One high MW polybutadiene was diluted with a low $M W(\approx 1800)$ polybutadiene. For these mixtures it is found that $\eta_{\mathrm{o}} \propto \phi^{3.35}$ and $G_{\mathrm{N}}^{\circ} \propto \phi^{2}$, where $\phi$ is the volume fraction of the high MW component. $J_{\mathrm{e}}^{\circ}$ of the mixtures depends somewhat more strongly on $\phi$ than according to $\phi^{-2}$. Both the MW and $\phi$ dependence of $\eta_{\mathrm{o}}$ and $J_{\mathrm{e}}^{\circ}$ are in agreement with Doi's modification of the reptation model that includes contour-length fluctuations. Experimental values of $J_{\mathrm{e}}^{\circ}$ are in quantitative agreement with the theory. The experimental loss $\left(G^{\prime \prime}\right)$, and storage $\left(G^{\prime}\right)$ moduli-frequency curves can be fitted with a high degree of precision to Doi's theory.

KEY WORDS Melt Rheology / Polybutadiene / Viscosity / Recoverable

Compliance / Doi-Edwards Theory / Contour-Length Fluctuation /
\end{abstract}

A long polymer chain in the melt or in a concentrated solution can be envisaged as confined to a tube. ${ }^{1}$ The walls of the tube consist of the neighbouring chains and are uncrossable. The motions of the chain perpendicular to the local tube axis are highly restricted, but the motion along the tube, called reptation, is possible. This simple model predicts that the zero-shear viscosity, $\eta_{\mathrm{o}}$, and $T_{\mathrm{d}}$ the longest relaxation time depend on $M^{3}$, that the recoverable compliance, $J_{\mathrm{e}}^{\circ}$, is independent of $M$, and that $J_{\mathrm{e}}^{\circ} \times G_{\mathrm{N}}^{\circ}=6 / 5\left(G_{\mathrm{N}}^{\circ}\right.$ is the plateau modulus). ${ }^{1,2}$ In fact, $\eta_{\mathrm{o}}, J_{\mathrm{e}}^{\circ}$, and $T_{\mathrm{d}}$ can all be derived from a knowledge of $G_{\mathrm{N}}^{\circ}$ and $M$ and two molecular parameters, the step length, $b$, and the monomeric friction coefficient, $\zeta_{0} \cdot{ }^{3}$ It has been shown that $G_{N}^{\circ}$ itself is also related to molecular parameters. ${ }^{4}$

Experimentally, however, the linear viscoelastic properties of high molecular weight linear polymers and their concentrated solutions exhibit more complex behaviour than the tube and reptation models predict. ${ }^{5,6}$ It has been pointed out that the tube model leads to an upper-limit theory for the viscoelastic properties applicable only at (unaccessibly) high molecular weight. ${ }^{3}$ Currently, tube renewal or constraint release ${ }^{7,8}$ and contour-length fluctuation $^{8,9}$ are considered as concurrent mechanisms for chain relaxation. These recent modifications attempt to bring the theoretical model into better agreement with experiment, in particular with the observed $\eta_{0} \propto M^{3.4}$ dependence and $J_{\mathrm{e}}^{\circ} \times G_{\mathrm{N}}^{\circ} \approx 2.0$.

The experimental $\eta_{\mathrm{o}} \propto M^{3.4}$ relation is now well established for numerous polymers. ${ }^{5,6}$ In this study we focussed our efforts on the experimental values of $J_{\mathrm{e}}^{\circ}$ and their dependence on molecular weight and concentration. Moreover the moduli-frequency curves of the samples were determined over a wide range of frequencies. These experimental results are compared with the predictions of the DoiEdwards theory ${ }^{2}$ and with Doi's modification 


\section{J. ROOVERS}

of this theory which includes contour-length fluctuations. ${ }^{9}$

For such comparison it is essential to work with monodisperse samples because $J_{\mathrm{e}}^{\circ}$ and the frequency dependence of $G^{\prime}$ and $G^{\prime \prime}$ are quite sensitive to the presence of even small amounts of non-uniformity. For example, experimental values of $J_{\mathrm{e}}^{\circ}$ for the much studied polystyrene melts increase slowly with increasing molecular weight. ${ }^{6,10,11}$ This is most likely due to a larger MW distribution in the high molecular weight samples. Also, the concentration dependence of $J_{\mathrm{e}}^{\circ}$ is variously found to be $\phi^{-2}$ or $\phi^{-2.2512}$ Polybutadiene was chosen here because its anionic polymerization yields very narrow MW distribution samples. Highly 1,4polybutadiene has a low value of $M_{\mathrm{e}}$, the molecular weight between entanglements, ${ }^{5}$ and $N=M / M_{\mathrm{e}}$, the number of entanglements per chain, can be varied over a wide range. Furthermore, rheological measurements can be made at room temperature. Care has to be taken, however, to avoid polymer degradation.

\section{EXPERIMENTAL}

The linear polybutadienes were prepared anionically in benzene with $s$ - BuLi at room temperature and terminated under vacuum with purified and degassed $t$-BuOH.${ }^{13}$ The polymers were precipitated in methanol containing 2,6-di- $t$-butyl-p-cresol as antioxidant, dried to constant weight and stored at $-20^{\circ} \mathrm{C}$ in vacuo in the dark.

The 1,2 unit content of the polybutadienes was determined from ${ }^{1} \mathrm{H}$ NMR spectra. It varies randomly between 8 and $11 \%$. The ratio of cis:trans units was determined from the intensities of the $27.4 \mathrm{ppm}$ (cis) and $32.7 \mathrm{ppm}$ (trans) signals in the ${ }^{13} \mathrm{C}$ NMR spectra $\left(\mathrm{CDCl}_{3}, \mathrm{TMS}\right.$ internal standard). The cis: trans ratio varies from 55/45 for PBD10 to 59/41 for PBD400. Number-average molecular weights were determined in toluene at $35^{\circ} \mathrm{C}$ with a Hewlett-Packard 503 high speed membrane osmometer. Weight-average molecular weights were measured in cyclohexane at $25^{\circ} \mathrm{C}$ with a Fica 50 photogoniometer. The Rayleigh ratio of benzene is $49 \times 10^{-6}$ for $\lambda_{\mathrm{o}}=436 \mathrm{~nm}$. The refractive index increment of polybutadiene in cyclohexane was redetermined with a Brice-Phoenix differential refractometer. It was found that $\mathrm{d} n / \mathrm{d} c=0.117_{5}$, in good agreement with the value from the literature, ${ }^{14}$ but $2.6 \%$ higher than our previous determination. ${ }^{15}$ The molecular weight of a few samples were also determined by light scattering in dioxane at $27^{\circ} \mathrm{C}$, a $\theta$-solvent. ${ }^{16}$ For this system $\mathrm{d} n / \mathrm{d} c=0.117_{2}$ for $436 \mathrm{~nm}$ light.

Intrinsic viscosities of the polymers were measured with Cannon-Ubbelohde viscometers in toluene at $35^{\circ} \mathrm{C}$. The intrinsic viscosities of selected samples were also measured in dioxane at $27^{\circ} \mathrm{C}$. It was checked with a variable shear viscometer that the intrinsic viscosities measured are shear-rate independent values.

The density of polybutadiene at $27^{\circ} \mathrm{C}$ $\rho_{\mathrm{o}}=0.89 \mathrm{~g} \mathrm{ml}^{-1} .{ }^{15}$ Mixtures of one high molecular weight linear polymer (PBD 165) with a low molecular weight polybutadiene $\left(M_{\mathrm{GPC}} \approx 1800\right)$ were made by weight. The two components were dissolved in benzene and the solutions evaporated to dryness under vacuo at room temperature. ${ }^{15}$

The viscoelastic properties of the polybutadienes and the mixtures were measured between parallel platens with a Rheometrics mechanical spectrometer in the oscillatory mode. The dynamic shear moduli, $G^{\prime}$ (storage) and $G^{\prime \prime}$ (loss), were measured at small strains in the linear regime for frequencies between 0.01 and $100 \mathrm{rad} \mathrm{s}^{-1}$. Five frequencies per decade were used. Measurements were made at a minimum of 6 temperatures between 106 and $-77^{\circ} \mathrm{C}$. The highest temperature is dictated by the need to obtain the zero-shear properties. The temperature control was provided by a stream of dry nitrogen. The rheological properties of all polymers, except the lowest one, were measured at $27^{\circ} \mathrm{C}$ and this temperature was used as the reference temperature $\left(T_{\mathrm{o}}\right)$. 
Storage and loss moduli master curves were constructed at the reference temperature with the data obtained at the other temperatures. Superposition of the moduli was achieved by first shifting the data vertically followed by a horizontal shift along the frequency axis. The vertical shift factors, $b_{\mathrm{T}}$, are approximately equal to $\left(\rho_{\mathrm{o}} T_{\mathrm{o}} / \rho T\right) .{ }^{15}$ The temperature dependence of the horizontal shift factors, $a_{\mathrm{T}}$, could be linearized by plotting $\log a_{\mathrm{T}}$ against $1 /\left(T-T_{\mathrm{oo}}\right)$ where $T_{\mathrm{oo}}=T_{\mathrm{g}}-60 . T_{\mathrm{g}}$ of polybutadiene is $-96^{\circ} \mathrm{C} .{ }^{15}$ The slopes of such plots are equal to $730 \pm 10 T$. $^{5}$

Examples of storage $\left(G^{\prime}(\omega)\right)$ and loss $\left(G^{\prime \prime}(\omega)\right)$ moduli-frequency master curves are shown in Figure 1. Master curves of the mixtures rich in the low molecular weight component require a small shift to take into account a small drop in $T_{\mathrm{g}}{ }^{15}$

The zero-shear viscosities are obtained from the low-frequency loss moduli according to ${ }^{5}$

$$
\eta_{0}=\lim _{\omega \rightarrow 0} \frac{G^{\prime \prime}(\omega)}{\omega}
$$

The zero-shear recoverable compliance is obtained according to ${ }^{5}$

$$
J_{\mathrm{e}}^{\circ}=\lim _{\omega \rightarrow 0} J^{\prime}(\omega)=\lim _{\omega \rightarrow 0} \frac{G^{\prime}(\omega)}{\left[G^{\prime \prime}(\omega)^{2}+G^{\prime}(\omega)^{2}\right]}
$$

In Figure 2 typical experimental data are shown from which the value of $J_{\mathrm{e}}^{\circ}$ is derived. The evaluation of $J_{\mathrm{e}}^{\circ}$ at the reference temperature can be aided by the experimental data obtained at other temperatures provided values of $J^{\prime}(\omega)$ are corrected by the appropriate $\left(\rho T / \rho_{\mathrm{o}} T_{\mathrm{o}}\right)$ factor. ${ }^{5}$ Values of $J_{\mathrm{e}}^{\circ}$ are reproducible to within $2 \%$.

The plateau moduli of the polybutadienes and their mixtures with low molecular weight polybutadiene are determined from ${ }^{5}$

$$
G_{\mathrm{N}}^{\circ}=\frac{2}{\pi} \int_{-\infty}^{+\infty}\left[G^{\prime \prime}(\omega)-G_{\mathrm{s}}^{\prime \prime}(\omega)\right] \mathrm{d} \ln \omega
$$

where $G_{\mathrm{s}}^{\prime \prime}(\omega)$ is the contribution of the transition zone to the loss moduli in the plateau region. This contribution is taken into account

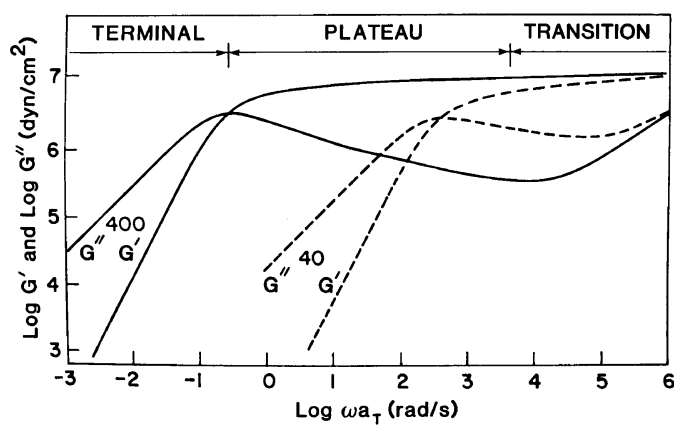

Figure 1. $\log -\log$ plot of the loss $\left(G^{\prime \prime}\right)$ and storage $\left(G^{\prime}\right)$ moduli against frequency for PBD40 and PBD400. The three different regions of the viscoelastic properties are for PBD400.

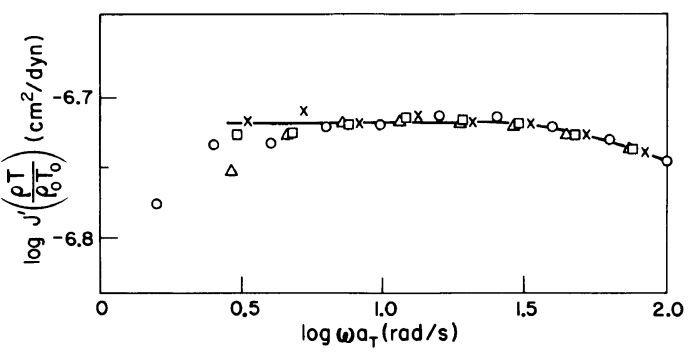

Figure 2. Evaluation fo $J_{\mathrm{e}}^{\circ}$ for PBD65. Data obtained at different temperatures. $\bigcirc, 27^{\circ} \mathrm{C} ; \triangle, 7^{\circ} \mathrm{C} ; \square$, $-12.5^{\circ} \mathrm{C} ; \times,-33^{\circ} \mathrm{C}$.

for frequencies larger than $4,000 \mathrm{rad} \mathrm{s}^{-1}$ $\left(\log \omega a_{\mathrm{T}}>3.6\right)$. The same values of $G_{\mathrm{s}}^{\prime \prime}(\omega)$ of reference 15 have been used. $G_{\mathrm{N}}^{\circ}$ cannot be determined with the same accuracy as $J_{\mathrm{e}}^{\circ}$, because shifted $G^{\prime \prime}(\omega)$ data have to be included. The subtraction and integration of eq 3 further increase the error margin.

\section{RESULTS}

The weight- and number-average molecular weights of the polybutadienes are given in Table I. The average value of $M_{w} / M_{n}=1.04$. It is felt that the real molecular weight distribution of the polymers is probably narrower. Indeed, the $M_{w} / M_{n}$ ratios from GPC, uncorrected for axial dispersion, are only about 1.05 , see Table I. No trend in the polydispersity with molecular weight can be discerned. 


\section{J. ROOVERS}

Table I. Characteristics of linear polybutadienes

\begin{tabular}{|c|c|c|c|c|c|c|c|}
\hline \multirow{2}{*}{\multicolumn{2}{|c|}{ Sample }} & \multirow{2}{*}{$M_{w} \times 10^{-5 a}$} & \multirow{2}{*}{$M_{n} \times 10^{-5 b}$} & \multicolumn{2}{|c|}{$M_{w}{ }^{\mathrm{c}} / M_{n}$} & \multirow{2}{*}{$\frac{[\eta]_{\mathrm{Tol}}^{35^{\circ} \mathrm{C}}}{\mathrm{dl} / \mathrm{g}^{-1}}$} & \multirow{2}{*}{$\frac{[\eta]_{\text {Diox }}^{27.5^{\circ} \mathrm{C}^{\mathrm{d}}}}{\mathrm{dl} / \mathrm{g}^{-1}}$} \\
\hline & & & & & (GPC) & & \\
\hline \multirow{11}{*}{ PBD } & 10 & $0.11_{1}$ & $0.10_{9}$ & 1.02 & - & $0.26_{9}$ & - \\
\hline & 20 & $0.23_{6}$ & $0.22_{7}$ & 1.04 & $1.05_{0}$ & $0.44_{0}$ & - \\
\hline & 39 & $0.39_{3}$ & $0.37_{9}$ & 1.04 & $1.05_{3}$ & $0.66_{2}$ & $0.39_{5}$ \\
\hline & 40 & $0.39_{4}{ }^{\mathrm{e}}$ & - & - & - & $0.65_{7}$ & - \\
\hline & 65 & 0.59 & $0.58_{2}$ & 1.01 & $1.04_{9}$ & $0.89_{2}$ & - \\
\hline & 120 & 1.14 & $1.09_{5}$ & 1.04 & $1.04_{8}$ & 1.40 & - \\
\hline & 165 & 1.55 & $1.47_{7}$ & 1.05 & $1.05_{0}$ & 1.77 & - \\
\hline & 170 & 1.64 & 1.52 & 1.08 & $1.05_{3}$ & 1.80 & - \\
\hline & 180 & 1.80 & - & - & - & 1.97 & 0.83 \\
\hline & 300 & 2.56 & 2.48 & 1.03 & $\left(1.06_{2}\right)^{\mathrm{g}}$ & 2.54 & - \\
\hline & 400 & $3.61^{f}$ & - & - & $\left(1.07_{2}\right)^{\mathrm{g}}$ & 3.23 & 1.22 \\
\hline
\end{tabular}

a Light scattering in toluene at $35^{\circ} \mathrm{C}$ with $\lambda=436$ and $\mathrm{d} n / \mathrm{d} c=0.117_{5}$.

b Osmotic Pressure in toluene $35^{\circ} \mathrm{C}$.

c Apparent from GPC without correction for zone spreading.

d $\theta$-Solvent.

e $M_{w}=0.387 \times 10^{5}$ in dioxane at $27^{\circ} \mathrm{C}$.

f $M_{w}=3.53 \times 10^{5}$ in dioxane at $27^{\circ} \mathrm{C}$.

g This value obtained by extrapolation of volume of elution-MW curve

Table II. Viscoelastic properties of linear polybutadienes at $300 \mathrm{~K}$

\begin{tabular}{|c|c|c|c|c|c|c|c|c|}
\hline \multicolumn{2}{|c|}{ Sample } & \multirow{2}{*}{$\frac{M_{w} \times 10^{-5}}{0.11_{1}}$} & \multirow{2}{*}{$\frac{N^{\mathrm{a}}}{5.63}$} & \multirow{2}{*}{$\frac{\eta^{\circ}}{2.25 \times 10^{2}}$} & \multirow{2}{*}{$\frac{J_{\mathrm{e}}^{\circ} \times 10^{7}}{1.55}$} & \multirow{2}{*}{$\frac{G_{\mathrm{N}}^{\circ} \times 10^{-7}}{0.99}$} & \multirow{2}{*}{$\frac{G_{\max }^{\prime \prime} \times 10^{-6}}{(3.04)^{\mathrm{b}}}$} & \multirow{2}{*}{$\frac{J_{\mathrm{e}}^{\circ} \times G_{\mathrm{N}}^{\circ}}{1.54}$} \\
\hline PBD & 10 & & & & & & & \\
\hline & 20 & $0.23_{6}$ & 12.0 & $2.82 \times 10^{3}$ & $1.79(1.76)^{\mathrm{c}}$ & 1.08 & 3.02 & 1.94 \\
\hline & 39 & $0.39_{3}$ & 20.0 & $1.77 \times 10^{4}$ & 1.99 & 1.05 & 2.76 & 2.09 \\
\hline & 40 & $0.39_{4}$ & 20.0 & $1.81 \times 10^{4}$ & 1.84 & 1.05 & 2.92 & 1.93 \\
\hline & 65 & 0.59 & 30.0 & $6.8 \times 10^{4}$ & 1.88 & 1.02 & 2.78 & 1.92 \\
\hline & 120 & 1.14 & 57.9 & $6.5 \times 10^{5}$ & $1.77(1.84)^{\mathrm{c}}$ & 1.08 & 3.05 & 1.97 \\
\hline & 165 & 1.55 & 78.7 & $1.8 \times 10^{6}$ & 1.77 & 1.07 & 3.02 & 1.89 \\
\hline & 170 & 1.64 & 83.3 & $2.1 \times 10^{6}$ & 1.64 & 1.16 & 3.28 & 1.90 \\
\hline & 180 & 1.80 & 97.4 & $3.1_{5} \times 10^{6}$ & 1.75 & 1.11 & 3.16 & 1.94 \\
\hline & 300 & 2.56 & 130.0 & $9.6 \times 10^{6}$ & 1.66 & 1.12 & 3.28 & 1.86 \\
\hline & 400 & 3.61 & 183.2 & $2.9 \times 10^{7}$ & 1.57 & 1.15 & 3.42 & 1.80 \\
\hline
\end{tabular}

The weight-average molecular weights obtained in dioxane agree with the cyclohexane data within experimental error. The weightaverage molecular weights in cyclohexane are retained as the principal characteristic of each sample.

The intrinsic viscosities of the polybutadienes in dioxane are well represented by $[\eta]_{\theta}=1.99 \times 10^{-3} M_{w}^{1 / 2}\left(\mathrm{dlg}^{-1}\right)$ in fair agreement with $[\eta]_{\theta}=1.78 \times 10^{-3} M_{w}^{1 / 2}$ given previously. ${ }^{16}$ The intrinsic viscosity data in toluene are described by $[\eta]=2.85 \times 10^{-4} M_{w}^{0.73}$ $\left(\mathrm{dl} \mathrm{g}^{-1}\right)$. This relation gives $[\eta]$ within $3 \%$ of those calculated with other relations for this polymer-solvent system. . $^{14,17}$

Values of the melt viscosities of the poly- 
Table III. Viscoelastic properties of solutions of polybutadienes at $300 \mathrm{~K}$

\begin{tabular}{ccccccc}
\hline \multirow{2}{*}{ Sample } & $\phi^{\mathrm{a}}$ & $M / M_{\mathrm{e}}{ }^{\mathrm{b}}$ & $\frac{\eta_{\mathrm{o}}}{2}$ & Poise & $\frac{J_{\mathrm{e}}^{\circ}}{\mathrm{cm}^{2} \mathrm{dyn}^{-1}}$ & $\frac{G_{\mathrm{N}}^{\circ}}{\mathrm{dyn} \mathrm{cm}^{-2}}$ \\
\hline \multirow{2}{*}{ PBD 165 } & 1.00 & 78.7 & $1.8 \times 10^{6}$ & $1.77 \times 10^{-7}$ & $1.07 \times 10^{7}$ \\
& 0.627 & 49.3 & $3.9 \times 10^{5}$ & $4.7 \times 10^{-7}$ & $4.5 \times 10^{6}$ \\
& 0.406 & 31.9 & $9.6 \times 10^{4}$ & $1.14 \times 10^{-6}$ & $2.0 \times 10^{6}$ \\
& 0.254 & 20.0 & $2.0 \times 10^{4}$ & $3.5 \times 10^{-6}$ & $6.8 \times 10^{5}$ \\
& 0.162 & 12.75 & $3.9 \times 10^{3}$ & $8.8 \times 10^{-6}$ & $2.8 \times 10^{5}$ \\
\hline
\end{tabular}

a Volume fraction of PBD165.

b Based on $M_{\mathrm{c}}=1970 / \phi$.

butadienes are given in Table II. The data are well represented by $\eta_{\mathrm{o}}=4.7 \times 10^{-12} M_{w}^{3.38}$. This is in fair agreement with $\eta_{\mathrm{o}}=5.2 \times$ $10^{-12} M_{w}^{3.4}$ at $24.5^{\circ} \mathrm{C}^{14}$ and $3.0 \times 10^{-12} M_{w}^{3.4}$ at $27^{\circ} \mathrm{C} .^{17}$ The discrepancy between these three $\eta_{\mathrm{o}}-M_{w}$ relation partly disappears when $[\eta]_{\text {Tol }}$ is substituted for $M_{w}$. The zeroshear viscosities of the mixtures of PBD165 with low molecular weight polybutadiene are given in Table III. They give $\eta_{\mathrm{o}} \propto \phi^{3.35}$.

The zero-shear recoverable compliances $J_{\mathrm{e}}^{\circ}$ of the polybutadiene melts are given in column 5 of Table II. It can be seen that the values of $J_{\mathrm{e}}^{\circ}$ go through a maximum at $M_{w} \approx 40,000$ and slowly decrease with increasing molecular weight. This is in contrast with the small increase or constant values of $J_{\mathrm{e}}^{\circ}$ usually found for narrow molecular weight linear entangled polymers. ${ }^{5,6,10,11}$ The zero-shear recoverable compliance for the solutions of PBD165 are given in Table III. Values of $J_{\mathrm{e}}^{\circ}$ decreases slightly faster than according to $\phi^{-2}$.

The plateau moduli of the melts, $G_{\mathrm{N}}^{\circ}$, are given in Table II. The high molecular weight values are in good agreement with those of the literature for this type of polybutadiene. ${ }^{5,6,14,18}$ The values agree also with those of four-arm star polybutadienes. ${ }^{15,18,19}$ It is not certain that the small decrease of $G_{\mathrm{N}}^{\circ}$ with decreasing molecular weight is real. The determination of $G_{\mathbf{N}}^{\circ}$ with eq 3 becomes progressively less accurate as the molecular weight of the sample decreases and the overlap between the plateau region and transition zone increases. The plateau moduli of the solutions of PBD165 are given in Table III. The data indicate that $G_{\mathrm{N}}^{\circ} \propto \phi^{2}$. A similar dependence was also found for stars diluted with low molecular weight polybutadiene. ${ }^{15}$ The result is in contrast with the $\phi^{2.25}$ dependence observed when polybutadiene is diluted with a low molecular weight, thermodynamically good solvent. ${ }^{18}$ The only study comparable with this work involved the dilution of polyisoprene with low molecular weight polyisoprene for which $J_{N}^{\circ}=\left(1 / G_{\mathrm{N}}^{\circ}\right) \propto \phi^{-2}$ was observed. ${ }^{20}$

In the last column of Table II the product $J_{\mathrm{e}}^{\circ} \times G_{\mathrm{N}}^{\circ}$ of each sample is listed. It can be seen that all products except one are less than 2.0 and some approach 1.8. Typical literature values of $J_{\mathrm{e}}^{\circ} \times G_{\mathrm{N}}^{\circ}$ are between 2.0 and 3.0. ${ }^{6,18,21,22}$ The product $J_{\mathrm{e}}^{\circ} \times G_{\mathrm{N}}^{\circ}$ attests to the narrow molecular weight distribution of the samples.

Values of $G_{\mathrm{N}}^{\circ}$ are used to calculate $M_{\mathrm{e}}$, the molecular weight between entanglements, according to 5

$$
M_{\mathrm{e}}=\frac{\phi \rho R T}{G_{\mathbf{N}}^{\circ}}
$$

With $G_{\mathrm{N}}^{\circ}=1.12 \times 10^{7} \mathrm{dyn}^{-2}$ for polybutadiene at $300 \mathrm{~K}, M_{\mathrm{e}}=1970 .{ }^{15}$ Since $G_{\mathrm{N}}^{\circ} \propto \phi^{2}$, it follows that $M_{\mathrm{e}}(\phi)=\phi^{-1} \times M_{\mathrm{e}}(\phi=1)$ for poly- 


\section{J. ROOVERS}

butadiene diluted with low molecular weight polymer.

\section{DISCUSSION}

The Doi-Edwards theory describes the relaxation of a deformed polymer chain in a matrix of virtually immobile neighbours., ${ }^{2,23}$ Three relaxation mechanisms, each specified by a characteristic relaxation time are considered. The shortest time process $\left(\tau_{\mathrm{A}}\right)$ involves equilibration of the chain over a distance " $a$ ", the mesh size or tube diameter. This process is independent of $M$ since

$$
\tau_{\mathrm{A}}=\frac{1}{6 \pi^{2}} \frac{\zeta_{0} b^{2}}{k T} N_{\mathrm{e}}^{2}
$$

where $\zeta_{\mathrm{o}}=1.1 \times 10^{-7} \mathrm{dyn} \mathrm{s} \mathrm{cm}^{-1},{ }^{19} b^{2}=4.306$ $\times 10^{-15} \mathrm{~cm}^{216}$ and $N_{\mathrm{e}}=\left(M_{\mathrm{e}} / M_{\mathrm{o}}\right)=36.4, \tau_{\mathrm{A}}=$ $2.56 \times 10^{-7} \mathrm{~s}$ for polybutadiene. This corresponds to $\log \omega a_{\mathrm{T}}=6.6$ which is well into the transition zone as can be seen from Figure 1.

The second process $\left(\tau_{\mathrm{B}}\right)$ is a relaxation whereby the contour length of the chain is restored to its equilibrium length. It involves movement of the chain along the tube. ${ }^{2} \tau_{B}$ is given $b y^{23}$

$$
\tau_{\mathrm{B}}=\frac{1}{3 \pi^{2}} \frac{\zeta_{0} b^{2}}{k T} N_{0}^{2}
$$

where $N_{\mathrm{o}}$ is the number of monomers along the chain. E.g., for PBD40 and PBD400 $\log \tau_{\mathrm{B}}=-3.70$ and -1.76 resp. Such times are located in the plateau region as can be seen in Figure 1. The complete disengagement from the original tube is by reptation. The longest relaxation time of the polymer, $T_{\mathrm{d}}$, is associated with this process according to ${ }^{2}$

$$
T_{\mathrm{d}}=\frac{1}{\pi^{2}} \frac{\zeta_{0} b^{2}}{k T} \frac{N_{0}^{3}}{N_{\mathrm{e}}}
$$

This characteristic time is found in the terminal region.

The moduli-frequency curves associated with the reptation mechanism are given by

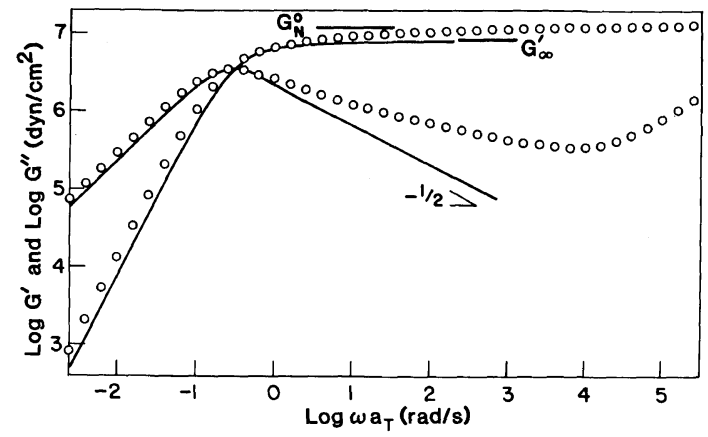

Figure 3. Comparison of the $G^{\prime \prime}(\omega)$ and $G^{\prime}(\omega)$ data (circles) of PBD 400 with the Doi-Edwards reptation model given by eq $8 \mathrm{a}$ and $8 \mathrm{~b}$. The curves are matched at the maximum in $G^{\prime \prime}(\omega)$.

$$
G^{\prime}(\omega)=\frac{\rho R T}{M_{\mathrm{e}}} \frac{8}{\pi^{2}} \sum_{n \text { odd }} \frac{1}{n^{2}} \frac{\omega^{2}\left(T_{\mathrm{d}} / n^{2}\right)^{2}}{1+\omega^{2}\left(T_{\mathrm{d}} / n^{2}\right)^{2}}
$$

and

$$
G^{\prime \prime}(\omega)=\frac{\rho R T}{M_{\mathrm{e}}} \frac{8}{\pi^{2}} \sum_{n \text { odd }} \frac{1}{n^{2}} \frac{\omega\left(T_{\mathrm{d}} / n^{2}\right)}{1+\omega^{2}\left(T_{\mathrm{d}} / n^{2}\right)^{2}}
$$

where $\left(\rho R T / M_{\mathrm{e}}\right)=G_{\mathrm{N}}^{\circ}$ from eq 4 .

The theoretical $G^{\prime}(\omega)$ and $G^{\prime \prime}(\omega)$ of eq 8 are compared with the experimental modulifrequency curves of PBD400 in Figure 3. The theoretical curve was matched to the experimental one at the maximum in $G^{\prime \prime}(\omega)$. A similar comparison was made for a single relaxation time. ${ }^{18}$ In general the experimental $G^{\prime}(\omega)$ and $G^{\prime \prime}(\omega)$ curves indicate a broader relaxation process than the Doi-Edwards theory predicts, both in the terminal and in the plateau zone. As a result, values of $J_{\mathbf{e}}^{\circ}$ are higher than predicted. By matching at the maximum in $G^{\prime \prime}(\omega)$ it is observed that $G_{\infty}^{\prime} \approx 0.70\left[G_{\mathrm{N}}^{\circ}\right]_{\exp }$ indicating that the terminal relaxation process of the Doi-Edwards theory accounts for about $70 \%$ of the total plateau modulus.

In the framework of the Doi-Edwards theory the part of the plateau modulus which is unaccounted for must originate in type- $B$ processes. However, the shape of the excess of $G^{\prime \prime}(\omega)$ in the plateau zone is not completely in 
agreement with a distinct secondary relaxation process. An analysis in those terms was recently attempted. ${ }^{24}$ Two additional relaxation processes were introduced to fit the model with the experiments. ${ }^{24}$ In any case, introduction of relaxation processes in the plateau region does not lead to a $\eta_{\mathrm{o}} \propto M^{3.4}$ dependence nor does it increase the theoretical $J_{\mathrm{e}}^{\circ}$ substantially. These short-comings have been pointed out. ${ }^{25}$

More recently Doi has introduced a model in which there is a continuous coupling between the reptation process and the contourlength fluctuations of the chain. ${ }^{9}$ The modulus at time $t$ after a sudden deformation is given by

$$
G(t)=\frac{\rho R T}{M_{\mathrm{e}}} \frac{2}{L_{\mathrm{eq}}} \int_{0}^{L_{\mathrm{eq}} / 2} \mathrm{~d} x P(t, x)
$$

where $P(t, x)$ is the probability that a tube segment at $x\left(0<x<L_{\mathrm{eq}} / 2, L_{\mathrm{eq}}\right.$ is the equilibrium length) has not been visited by its nearest chain end at time $t$, and is therefore still in the deformed state.

Without contour-length fluctuations the model predicts

$$
G(t)=\frac{\rho R T}{M_{\mathrm{e}}} \int_{0}^{1} d \xi \exp \left(\frac{-t}{\tau_{\xi}}\right)
$$

where

$$
\tau_{\xi}=\left(\frac{2 x}{L_{\mathrm{eq}}}\right)^{2} T_{\mathrm{d}}^{\infty}, \xi=\frac{2 x}{L_{\mathrm{eq}}}, \quad \text { and } \quad T_{\mathrm{d}}^{\infty}=\tau_{L_{\mathrm{eq}} / 2}
$$

Also

$$
\eta_{0}=\frac{1}{3} G_{\mathrm{N}}^{\circ} T_{\mathrm{d}}^{\infty}
$$

and

$$
J_{\mathrm{e}}^{\circ} \times G_{\mathrm{N}}^{\circ}=9 / 5
$$

When fluctuations are included, $\tau_{\xi}$ has two types of behaviour. For a tube segment less than $\sqrt{N_{\mathrm{o}}} \cdot b$ from the chain end, the relaxation will be fast of the order of $T_{\text {eq }}$ of eq 6 and

$$
\tau_{\xi}(1)=\frac{N \xi^{4}}{16} T_{\mathrm{d}}^{\infty} \quad \xi \leqslant \frac{2}{\sqrt{N}}
$$

For tube segments farther from the chain-end,

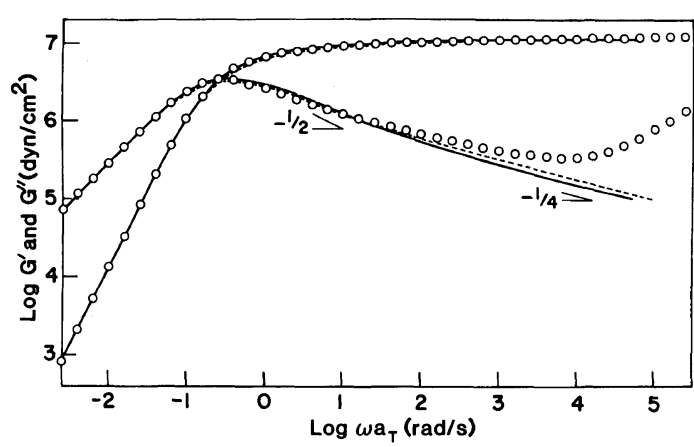

Figure 4. Comparison of the $G^{\prime \prime}(\omega)$ and $G^{\prime}(\omega)$ data (circles) of PBD400 with Doi's modification of the reptation model. Full lines eq. $14 \mathrm{a}$ and $14 \mathrm{~b}$ with eq $13 \mathrm{a}$ and 13b. Dotted lines calculated with the improved coefficient (1.47). See the text. The curves are matched vertically with the experimental $G_{\mathrm{N}}^{\circ}$ and horizontally with $\eta^{\circ}$.

the relaxation time is given by

$$
\tau_{\xi}(2)=(\xi-1 / \sqrt{N})^{2} T_{\mathrm{d}}^{\infty} \quad 1 \geqslant \xi \geqslant \frac{2}{\sqrt{N}}
$$

Equations $13 \mathrm{a}$ and $13 \mathrm{~b}$ represent Doi's smoothed version. ${ }^{9}$ The time dependence of the modulus eq 10 is now given by the sum of two integrals and

$$
\begin{aligned}
G^{\prime}(\omega)= & \frac{\rho R T}{M_{\mathrm{e}}} \int_{0}^{2 / \sqrt{N}} \frac{\omega^{2}\left(\tau_{\xi}(1)\right)^{2}}{1+\omega^{2}\left(\tau_{\xi}(1)\right)^{2}} \\
& +\int_{2 / \sqrt{N}}^{1} \frac{\omega^{2}\left(\tau_{\xi}(2)\right)^{2}}{1+\omega^{2}\left(\tau_{\xi}(2)\right)^{2}}
\end{aligned}
$$

and

$$
\begin{aligned}
G^{\prime \prime}(\omega)= & \frac{\rho R T}{M_{\mathrm{e}}} \int_{0}^{2 / \sqrt{N}} \frac{\omega \tau_{\xi}(1)}{1+\omega^{2}\left(\tau_{\xi}(1)\right)^{2}} \\
& +\int_{2 / \sqrt{N}}^{1} \frac{\omega \tau_{\xi}(2)}{1+\omega^{2}\left(\tau_{\xi}(2)\right)^{2}}
\end{aligned}
$$

In that case

$$
\eta_{0}=\frac{1}{3} G_{\mathrm{N}}^{\circ} T_{\mathrm{d}}^{\infty}\left[(1-1 / \sqrt{N})^{3}+\frac{1}{5 N^{2} \sqrt{N}}\right]
$$

and 


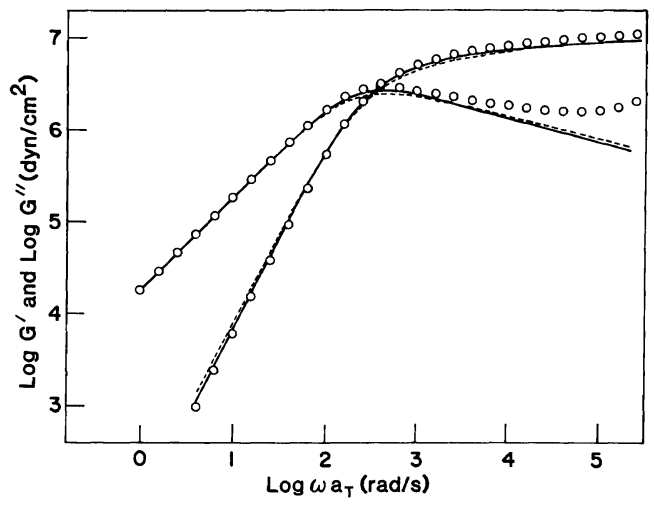

Figure 5. Same as Figure 4 for PBD40.

$$
J_{\mathrm{e}}^{\circ}=\frac{9}{5 G_{N}^{\circ}} \frac{(1-1 / \sqrt{N})^{5}+\frac{1}{9 N^{2} \sqrt{N}}}{\left[(1-1 / \sqrt{N})^{3}+\frac{1}{5 N \sqrt{N}}\right]^{2}}
$$

In Figure 4 the experimental $G^{\prime}(\omega)$ and $G^{\prime \prime}(\omega)$ curves of PBD400 are compared with those calculated with eq $14 \mathrm{a}$ and $14 \mathrm{~b}$ with $\tau_{\xi}$ given by eq 13a and 13b. The theoretical curves were superimposed on the experimental ones by shifting vertically to match $\rho R T / M_{\mathrm{e}}$ with the experimental $G_{\mathrm{N}}^{\circ}$ of each polymer and shifting horizontally until the terminal regions of $G^{\prime \prime}(\omega)$ coincided $\left(\eta_{\mathrm{o}}\right)$. It can be seen that the calculated moduli frequency curves closely fit the experimental curves. In particular the terminal region of $G^{\prime}(\omega)$ is in good agreement, indicating that with the inclusion of contourlength fluctuation a numerically correct value of $J_{\mathrm{e}}^{\circ}$ can be calculated. In the plateau region $G^{\prime \prime}$ depends on $\omega^{-0.4}$ at frequencies just larger than the maximum (theoretically -0.5 ). At high frequencies $G^{\prime \prime}$ depends on $\omega^{-0.15}$ (theoretically -0.25$).{ }^{9}$ The agreement is acceptable in view of the narrow frequency range for each dependence and the necessary transition zones between them. Introduction of Doi's improved 1.47 coefficient $^{9}$ introduces a marginal improvement in fitting theory with the experimental data. In Figure 5 the same comparison is shown for the low MW PBD40. Inclusion of contour-length fluctuations cor-

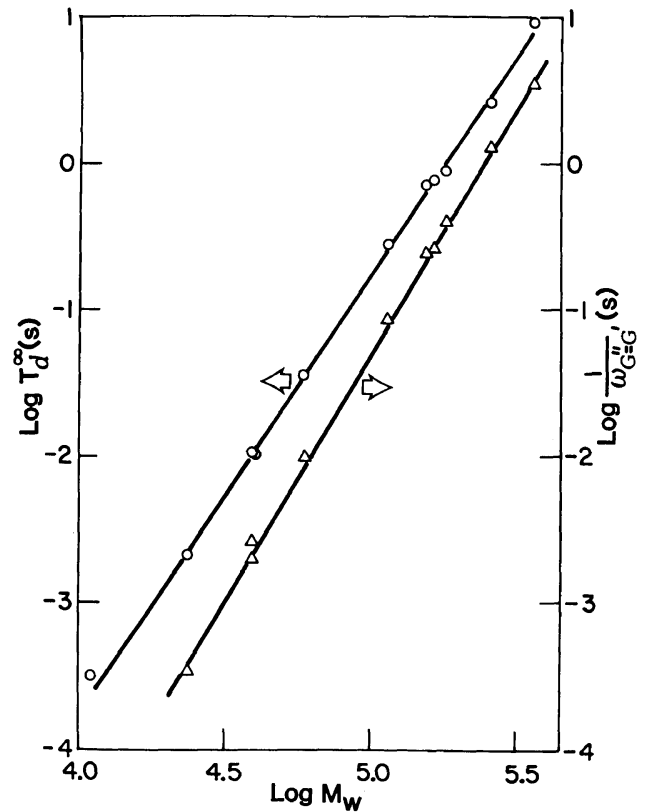

Figure 6. Circles: Log-log plot of $T_{\mathrm{d}}^{\infty}$ vs. MW. $T_{\mathrm{d}}^{\infty}$ from eq 15. Triangles: $\log -\log$ plot of $1 / \omega_{G^{\prime \prime}}=G^{\prime}$ i.e., the approximate experimental longest relaxation time $v s$. MW.

rectly predicts that $G_{\max }^{\prime \prime}$ decreases with decreasing number of entanglements. (Table II). From Figure 5 it can be seen that there are small discrepancies between experiment and theory for $G^{\prime}$ in the terminal zone and for $G^{\prime \prime}$ at the maximum, which indicate that the experimental relaxation time spectrum is slightly narrower than the theoretical one.

Comparisons of experimental and calculated $G^{\prime}(\omega)$ and $G^{\prime \prime}(\omega)$ curves were made for the other melts and for the mixtures with PBD165 and agreement similar to that shown for PBD400 were obtained. For PBD10 and 20 the trends observed in the moduli-frequency curves of PBD40 are further accentuated.

In Figure $6 T_{\mathrm{d}}^{\infty}$ calculated from eq 15 with the experimental values of $\eta_{\mathrm{o}}$ and $G_{\mathrm{N}}^{\circ}$ are shown as a function of molecular weight. It is found that $T_{\mathrm{d}}^{\infty} \propto M^{3.0}$ as theoretically expected. The values of $T_{d}^{\infty}$ are in quantitative agreement with $T_{\mathrm{d}}$ from eq 7 with the molecular constants given for eq 5 . This agreement 


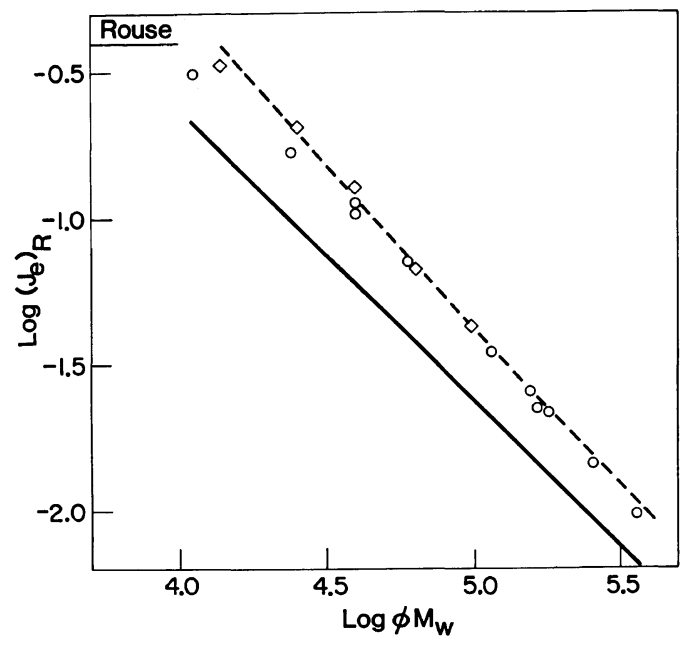

Figure 7. Double logarithmic plot of the reduced compliance $\left(J_{\mathrm{e}}\right)_{\mathrm{R}}$ (eq 18) against the product $\phi \cdot M_{w}$. $\bigcirc$, melts; $\square$, dilutions of PBD165; — - Doi-Edwards theory; --- , Doi's theory with chain-end fluctuation eq 16 . The horizontal line marked Rouse is $\left(J_{e}\right)_{R}=0.40$ of the Rouse theory for the isolated linear chain.

must be considered fortuitous because $T_{\mathrm{d}}^{\infty}$ is expected to be at least 4 time smaller than $T_{\mathrm{d}}$. Figure 6 also shows the molecular weight dependence of $1 / \omega_{G^{\prime \prime}=G^{\prime}}$ where $\omega_{G^{\prime \prime}=G^{\prime}}$ is the frequency of cross-over of $G^{\prime \prime}$ and $G^{\prime}$ in the terminal region. For linear polymers $1 / \omega_{G^{\prime \prime}}=G^{\prime}$ is approximately equal to the longest relaxation time of the polymer. The difference between $T_{\mathrm{d}}^{\infty}$ and $1 / \omega_{G^{\prime \prime}=G^{\prime}}$ is then due to contour-length fluctuation.

In Figure 7 the experimental values of $J_{\mathrm{e}}^{\circ}$ are compared with those calculated with eq 16 . In order to compare melt and diluted polymer samples the reduced compliance ${ }^{4}$ is used

$$
\left(J_{\mathrm{e}}\right)_{\mathrm{R}}=J_{\mathrm{e}}^{\circ} \frac{M}{\rho \phi R T}=J_{\mathrm{e}}^{\circ} \frac{G_{\mathrm{N}}^{\circ}}{N}
$$

It can be seen that Doi's eq 16 describes well the molecular weight and concentration dependence of $J_{\mathrm{e}}^{\circ}$. A similar equation in which $1 / \sqrt{N}$ is replaced by $1.47 / \sqrt{N}$ to correspond with Doi's more refined model gives $\left(J_{\mathrm{e}}\right)_{\mathrm{R}}$ slightly larger than the experimental values.

\section{CONCLUSION}

The complete moduli-frequency curves of 11 samples of entangled linear polybutadienes melts and 5 dilutions of one linear polymer with low MW polybutadiene have been used to evaluate the Doi-Edwards theory of viscoelasticity and Doi's theory which includes contour-length fluctuations. The former theory gives a rather poor agreement with experiment. The latter theory correctly predicts the $\eta_{\mathrm{o}}$ and $J_{\mathrm{e}}^{\circ}$ dependences on MW and concentration. The general shape of the $G^{\prime \prime}(\omega)$ and $G^{\prime}(\omega)$ curves is also well represented by the latter theory. It is experimentally observed, in agreement with the contour-length fluctuation hypothesis, that $G^{\prime \prime}(\omega)$ and $G^{\prime}(\omega)$ curves for narrow MW distribution linear polymers depend on $N$ the number of entanglements per molecule.

It should be noted that the major improvement in fitting the experimental $G^{\prime}(\omega)$ and $G^{\prime \prime}(\omega)$ curves with theory is observed when the Doi-Edwards theory with its set of discrete relaxation times (eq $8 \mathrm{a}$ and $8 \mathrm{~b}$ ) is abandoned for a model with a continuous spectrum of relaxation times (eq 9). In using the continuous spectrum, the distribution of relaxation times widens and $J_{\mathrm{e}}^{\circ} \propto G_{\mathrm{N}}^{\circ}$ increases to $9 / 5$ from $6 / 5$ in the Doi-Edwards theory. Introduction of contour-length fluctuations produces a minor correction to the continuous spectrum of relaxation times and leads to the molecular weight and concentration dependence of $\eta_{0}$ and $J_{\mathrm{e}}^{\circ}$ in close agreement with experiment. Models with a continuous spectrum of relaxation times are also used to describe the viscoelastic properties of regular stars. ${ }^{26,27}$

In the comparison of the theories with experiment constraint release was not included. This relaxation mechanism is expected to influence the viscoelastic properties of low MW entangled polymers. ${ }^{7}$ There is experimental evidence that the effect of constraint release dies out when the linear homopolymer chains are more than about 10 entanglement 


\section{J. ROOVERS}

lengths. ${ }^{28,29}$ This agrees with the observation that Doi's theory becomes poor for PBD10 and PBD20.

Acknowledgement. The author thanks Mr. P. M. Toporowski for help with the characterization of the samples. Issued as NRCC No. 25286.

\section{REFERENCES}

1. P.-G. de Gennes, J. Chem. Phys., 55, 572 (1971).

2. M. Doi and S. F. Edwards, J. Chem. Soc., Faraday Trans., 74, 1818 (1978).

3. W. W. Graessley, J. Polym. Sci., Polym. Phys. Ed., 18, 27 (1980).

4. W. W. Graessley and S. F. Edwards, Polymer, 22, 1329 (1981).

5. J. D. Ferry, "Viscoelastic Properties of Polymers," 3rd ed, John Wiley, N. Y. (1980).

6. W. W. Graessley, Adv. Polym. Sci., 16, 1 (1974).

7. J. Klein, Macromolecules, 11, 852 (1978).

8. W. W. Graessley, Adv. Polym. Sci., 47, 67 (1982).

9. M. Doi, J. Polym. Sci., Polym. Lett. Ed., 19, 265 (1981); Polym. Phys. Ed., 21, 667 (1983).

10. S. Onogi, T. Masuda, and K. Kitagawa, Macromolecules, 3, 109 (1970).

11. J. P. Monfort, G. Marin, and P. Monge, Macromolecules, 17, 1551 (1984).

12. W. W. Graessley, Royal Soc. Faraday Div., Faraday Symp., 18, 1 (1983).
13. D. J. Worsfold and S. Bywater, Macromolecules, 11, 582 (1978).

14. W. E. Rochefort, G. G. Smith, H. Rachapudy, V. R. Raju, and W. W. Graessley, J. Polym. Sci., Polym. Phys. Ed., 17, 1197 (1979).

15. N. Hadjichristidis and J. Roovers, Polymer, 26, 1087 (1985).

16. N. Hadjichristidis, Xu Zhongde, L. J. Fetters, and J. Roovers, J. Polym. Sci., Polym. Phys. Ed., 20, 743 (1982).

17. J. T. Gruver and G. Kraus, J. Polym. Sci., A, 2, 797 (1964).

18. V. R. Raju, E. V. Menezes, G. Marin, W. W. Graessley and L. J. Fetters, Macromolecules, 14, 1668 (1981).

19. J. Roovers, Polymer, 26, 1091 (1985).

20. N. Nemoto, T. Ogawa, H. Odani, and M. Kurata, Macromolecules 5, 641 (1972).

21. J. T. Gotro and W. W. Graessley, Macromolecules, 17, 2767 (1984).

22. J. M. Carella, W. W. Graessley, and L. J. Fetters, Macromolecules, 17, 2775 (1984).

23. M. Doi, J. Polym. Sci., Polym. Phys. Ed., 18, 1005 (1980).

24. Y.-H. Lin, Macromolecules, 17, 2846 (1984).

25. K. Osaki and M. Doi, Polym. Eng, Rev., 4, 35 (1984).

26. M. Doi and N. Y. Kuzuu, J. Polym. Sci., Polym. Lett. Ed., 18, 775 (1980)

27. D. S. Pearson and E. Helfand, Macromolecules, 17, 888 (1984).

28. J.-P. Montfort, G. Marin, and P. Monge, Macromolecules, 17, 1551 (1984).

29. J. Roovers, unpublished results. 